\title{
Quantitative Processing of EDS Maps: A Presentation of Solutions to Mapping Artifacts and Applications in Cosmochemistry
}

\author{
L. Kööp $p^{1,2}$ and A. M. Davis ${ }^{1,2,3}$ \\ 1. Department of the Geophysical Sciences, University of Chicago, Chicago, IL, USA \\ 2. Chicago Center for Cosmochemistry, Chicago, IL, USA \\ 3. Enrico Fermi Institute, University of Chicago, Chicago, IL, USA
}

X-ray mapping is widely used to study the abundance and distribution of elements in solids. With the arrival of large-area silicon drift detectors, large amounts of chemical data can be collected on fairly short timescales. Many samples of interest (e.g., rocks) contain imperfections (e.g., voids, relief) that can introduce mapping artifacts. To minimize the effect of artifacts, it is useful to produce unnormalized maps and reject analyses from spots that have totals deviating from $100 \%$ by more than a defined threshold. However, this requires that constant beam currents and working distances are maintained, as well as appropriate beam measurement standards and exact knowledge of the thickness of conductive coatings. Often, these conditions cannot be met, and it is useful to normalize each point analysis in a map to $100 \%$. In such cases, deviations from $100 \%$ cannot be used for internal quality control.

Here, we present examples of mapping artifacts and a quantitative analysis procedure for large chemical mapping datasets. We use two refractory inclusions from the Murchison meteorite (calcium-aluminumrich inclusions (CAIs) 2-2-8 and 1-6-7) as examples. CAI 2-2-8 is a zoned single hibonite $\left(\mathrm{CaAl}_{12} \mathrm{O}_{19}\right)$ crystal with abundant holes filled with epoxy (Fig. 1). CAI 1-6-7 is an aggregate of spinel $\left(\mathrm{MgAl}_{2} \mathrm{O}_{4}\right)$, gehlenite $\left(\mathrm{Ca}_{2} \mathrm{Al}_{2} \mathrm{SiO}_{7}\right)$ and hibonite (Fig. 2), with multiple cavities (some of which some are filled with epoxy). Both CAIs have been previously presented in [1,2] and the figures have been presented in [2].

The x-ray maps were collected using a Tescan LYRA3 FIB-SEM equipped with two Oxford XMax SDD EDS detectors. The 'raw' data were exported from the Oxford operating software. For each element of interest, both raw counts and normalized concentration files are used. For the coating element (here: C), we only use the raw counts file. To process the chemical datasets efficiently, we use the Python library pandas. Below, we summarize the main steps of our data analysis routine. If desired, each step can be performed independently of each other. Figures of maps and data files can be exported for each step.

Step 1: Filtering out spot analyses collected from (a) the embedding material and/or (b) uneven surfaces. For (a) we use a raw counts map of an element that is abundant in the embedding material, but rare in the sample (here, we use $\mathrm{C}$, as the CAIs are cast in epoxy) and reject analyses from spots that have $\mathrm{C}$ counts above a chosen number (here, we rejected analyses with $\mathrm{C}>400 \mathrm{cts}$ ). For (b), spot analyses are rejected for which the most abundant element shows unusually low counts at a given concentration. A comparison of Figs. $1 b$ \& $1 c$ as well as $2 b \& 2 c$ shows that this step efficiently removes spot analyses of epoxy and depressions in the sample. Step 2: Filtering out data with high relative uncertainties. For each element in a spot analysis, relative uncertainties are calculated based on counting statistics. Here, we rejected concentrations with relative uncertainties $>20 \%$. Step 3: Rejection of spot analyses with low totals. This step should be performed for unnormalized maps and can also be useful for normalized maps if step 2 has been performed. Here, we rejected spot analyses with totals deviating from $100 \mathrm{wt} \%$ by $3.0 \mathrm{wt} \%$ or more. Additional steps: We have also implemented algorithms that produce RGB images for any chosen set of three elements (Fig. 2d) and color-coded phase maps. Furthermore, we can calculate formulae for selected 
minerals. Such data can be used to assess the chemical variation in minerals (Fig. $2 \mathrm{~g}$ ). Here, we find that hibonite compositions are more variable in CAI 2-2-8 than in CAI 1-6-7 (Fig. 2g).

The advantages of our quantitative processing are shown in Figs. $1 \& 2$. In the unprocessed $\mathrm{Al}_{2} \mathrm{O}_{3}$ and $\mathrm{SiO}_{2}$ maps, epoxy-filled holes seem to increase the range of concentrations in the hibonite grain (Figs. 1b $\& \mathrm{~d})$. In the processed maps, the holes are excluded and the maps show a more appropriate $\mathrm{Al}_{2} \mathrm{O}_{3}$ zoning that is complemented by zoning in $\mathrm{MgO}$ and $\mathrm{TiO}_{2}$ (compare Fig. $2 \mathrm{~g}$ ). The importance of step 2 is shown in Fig. 2. The unprocessed map (Fig. 2e) suggests that FeO is present in low concentrations and is approximately uniformly distributed between hibonite, spinel and gehlenite. The processing (Fig 2f) reveals that $\mathrm{FeO}$ abundances in these primary minerals are associated with high uncertainties. Significant amounts of $\mathrm{FeO}$ only appear to be present in alteration phases at the edges of the inclusion.

In summary, quantitative processing improves the quality of x-ray maps and removes artifacts effectively. It also allows exporting and analyzing the processed data for individual minerals, which is useful to compare the range of chemical variation between samples.

\section{References:}

[1] L. Kööp et al. Geochimica et Cosmochimica Acta 184 (2016), 151.

[2] L. Kööp and A. M. Davis, Lunar \& Planetary Science 46 (2017) \#2136.
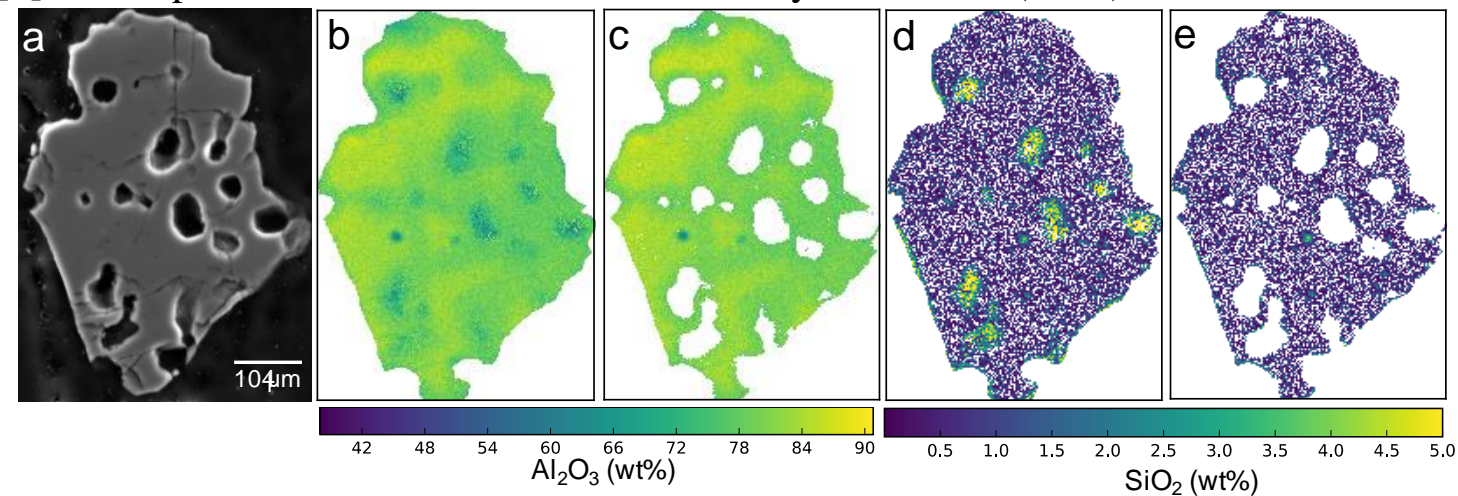

Figure 1. a) SE image of the CAI 2-2-8 showing holes filled with epoxy. $\mathrm{Al}_{2} \mathrm{O}_{3}$ (b, c) and $\mathrm{SiO}_{2}$ (d, e) maps are shown. b) and d) are before, c) and e) are after quantitative processing.
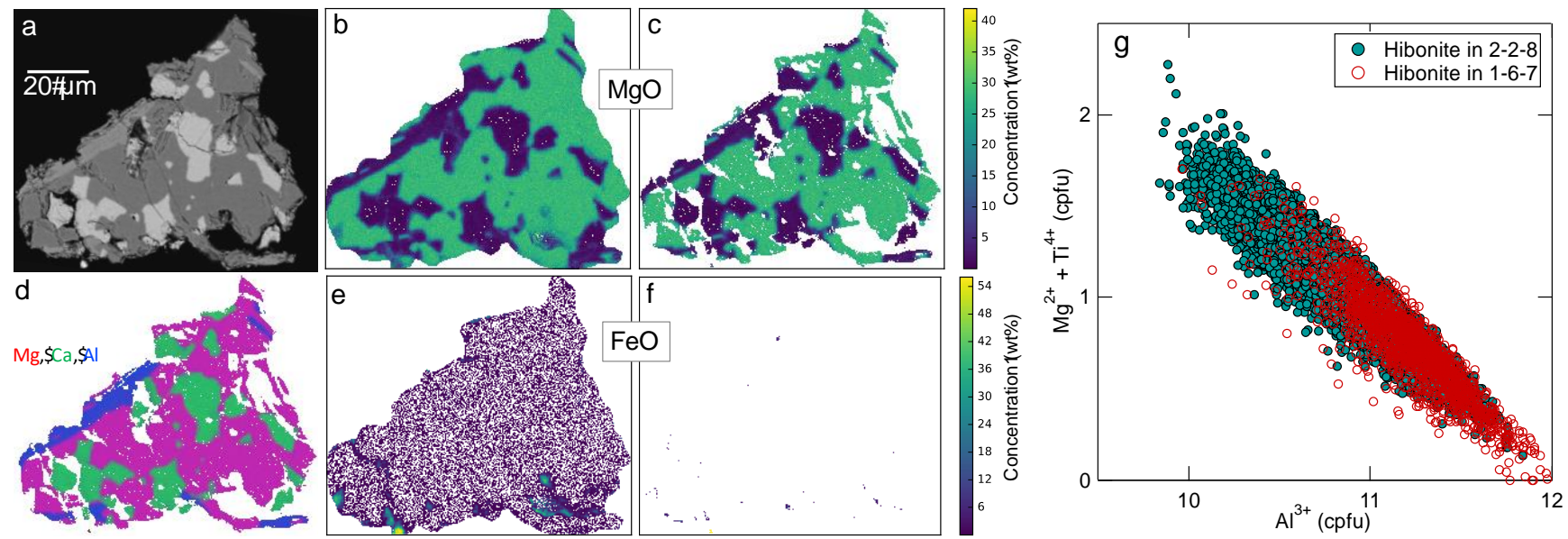

Figure 2. a) Backscattered electron image of the CAI 1-6-7 (from [1], modified). $\mathrm{MgO}$ (b, c) and $\mathrm{FeO}(\mathrm{e}, \mathrm{f}$ ) maps are shown. b) and e) are before, c) and f) are after quantitative processing. d) RGB image. g) Comparison of compositions measured in the mineral hibonite in CAIs 2-2-8 and 1-6-7. 